\title{
Primary Fallopian Tube Carcinoma (PFTC), A Rare Malignancy of Female Genital Tract
}

\author{
FB RASHID $^{\mathrm{a}}$, MAK AZAD
}

\begin{abstract}
Summary:
Background: Primary fallopian tube carcinoma (PFTC) is one of the rarest malignancies of female genital tract. It represents $<1 \%$ of all gynecologic malignancies. Preoperative diagnosis is uncommon due to its rarity and non-specific symptoms. In most cases diagnosis is made during surgery or histological examination. Rarity of this type of carcinoma prompted us to report it as individual case. Case: A 40-yearold parous women presented with bilateral PFTC. The patient gave a history of lower abdominal and pelvic pain for 2 years on several occasions. An abdominal ultrasound finding showed an adnexal mass and her CA125 level was 30IU/ml (normal- $<35 I U / m l)$. Clinically she was suspected as a case of pelvic inflammatory disease (PID). She underwent Total
\end{abstract}

\section{Introduction:}

Primary fallopian tube carcinoma is rare. It accounts for approximately for $0.14-1.8 \%$ of the female genital malignancy ${ }^{1}$.The fallopian tube is frequently involved secondarily with malignancy from other primary sites. Secondary malignant lesions usually arise from the adjacent ovary or uterus, occasionally from the gastrointestinal tract and rarely from the breast. They may also be involved in primary peritoneal carcinomatosis ${ }^{2}$. Incidence of primary carcinoma varies. In Western countries, it usually represents about $1 \%$ of all female genital malignancies ${ }^{3}$.

The etiology is unknown. It is thought to be associated with chronic tubal inflammation, infertility, tuberculous salpingitis and tubal endometriosis. Like ovarian carcinoma a BRCA gene mutation is associated with fallopian tube malignancy ${ }^{4}$. Clinical symptoms and signs are non-specific and include lower abdominal and pelvic pain, vaginal discharge and pelvic mass. A malignancy arising from the fallopian tube can be misdiagnosed as

a. Dr. Farhana Binte Rashid, Consultant, Kurmitola General Hospital, Dhaka.

b. Dr. Mohammad Abul kalam Azad, Medical officer, Department of Rheumatology, Bangabandhu Sheikh Mujib Medical University, Dhaka

Address of Correspondence: Dr. Farhan Binty Rashid, Junior Consultant, Kurmitola general Hospital, Mobile -01914424000, E Mail-Farhana.nuha32@gmail.com
Abdominal Hysterectomy with bilateral salpingoophorectomy. Intraoperative findings were consistent with PID. Final pathologic analysis showed bilateral primary fallopian tube carcinoma-well differentiated serous adenocarcinoma. Post operatively she was referred for oncological management. Conclusion: Malignancy should be considered in the differential diagnosis of PID especially in premenopausal age and intraoperative frozen section biopsy is crucial to make correct diagnosis and to allow appropriate surgical staging.

Key words: Primary fallopian tube carcinoma, Adenocarcinoma

(J Bangladesh Coll Phys Surg 2020; 38: 49-52)

DOI: http://dx.doi.org/10.3329/jbcps.v38i1.44689

ovarian tumor or tubo-ovarian mass. The diagnosis of primary fallopian tube is rarely considered preoperatively and in most cases it is an intraoperative findings or histological diagnosis. Regarding imaging studies ultrasound, the simplest imaging modality, is first to be performed. It helps to detect adnexal masses although it is often nonspecific in its finding. But preoperative work up with MRI can improve diagnosis for non-specific adnexal mass. MRI is the modality of choice for evaluating an undetermined pelvic mass on ultrasound $^{5}$. In a study Ma et al. showed the use of MRI for differentiating PFTC from epithelial ovarian cancer (EOC). According to their assessment of MRI features, PFTC often appears as a small-sized solid mass, with a sausage-like shape, homogeneous signal, mild or moderate enhancement, hydrosalpinx, or intrauterine fluid accumulation. So, MRI can identify the characteristic features of PFTC and differentiate PFTC from $\mathrm{EOC}^{6}$. For diagnosis of primary tubal carcinoma, both the ovaries and the uterus should appear normal on gross examination. The tube should be grossly abnormal resembling chronic salpingitis. The diagnosis is made histologically based on identifying a malignant change involving the line of fallopian tube.

Case report:

A 40-year-old woman $\mathrm{P}_{2+2}$ presented to Kurmitola General Hospital, Dhaka in November of, 2017 with the 
complaints of pain in lower abdomen and fever for the previous 7 days. Clinically an UTI was suspected. She had a previous history of PID diagnosed in 2015. An ultrasound scan showed a right- sided hydrosalpinx and was treated with antibiotics.

In March of 2016 she was hospitalized with severe lower abdominal pain. A transvaginal sonography showed two elongated cystic areas in both adnexal regions measuring about $4.3 \times 1.2 \mathrm{~cm}$ on right and $2.7 \times 1.5 \mathrm{~cm}$ on left ovary. Further evaluation was recommended but she was lost to follow up.

She had regular menstrual cycles and she did not practice any contraception. Her two deliveries were spontaneous vaginal deliveries and her last child was 14 years old. She was non-diabetic, normotensive, but a patient of chronic bronchial asthma, controlled with medications. She was also a carrier of hepatitis B virus (HBV) for last one and half years. Her personal and family histories were unremarkable and she had no history of gynecologic malignancy in the family. Her bimanual examination revealed bulky, anteverted, mobile uterus with, all fornices free and non-tender. No mass could be felt through any of the fornices.

Her transvaginal sonogram showed normal sized uterus with endometrial calcification and a cystic area measuring about $(8.5 \times 5.0) \mathrm{cm}$ containing solid component in the left adnexal region. Her CA 125 antigen level was $30 \mathrm{IU} / \mathrm{ml}$. Cervical cytology was negative for intraepithelial lesions or malignancy.

The patient was empirically treated with antibiotics for a presumptive diagnosis of PID. Her pain and fever subsided. She then underwent an exploratory laparotomy. Grossly, there was hydrosalpinx on left side with severe adhesion between both adnexa and uterus. Uterus and both ovaries were normal in size and apparently healthy looking. Her intraoperative diagnosis was pelvic inflammatory disease and a total abdominal hysterectomy with bilateral salpingo-oophorectomy was performed. The specimen was sent for histopahological analysis.

The final histopathologic diagnosis was well differentiated primary serous adenocarcinoma of both fallopian tubes. Ovarian surface involvement was absent in both sides on gross examination but microscopically one ovary was involved. Both cervix and uterus were free from tumor. [Pathologic staging: mpTNM. Primary tumor-mpT2a (Extension or metastasis to the uterus and ovaries.) Regional lymph node-mpNx. Distant metastasis- mpMx ] - Not clear what does it mean.

Seven days after surgery the patient was discharged and referred to an oncologist.

The following segment can be removed from the write up.

(Histopathology Report: Gross Description Specimen: Uterus with cervix with both sided adnexa, Specimen integrity: Single intact specimen. Both ovarian capsules intact, Primary tumor site: Both fallopian tubes, Tumor location: Fimbria, Tumor size: One fallopian tube Greatest dimension- $16 \mathrm{~mm}$, Additional dimension$(08 \times 12) \mathrm{mm}$, Other fallopian tube- Greatest dimension$10 \mathrm{~mm}$, Additional dimension- $(10 \times 08) \mathrm{mm}$, Cut surface: Cut surface of one ovary shows a grayish white area measuring, $(10 \times 08) \mathrm{mm}$ in maximum diameters.

Microscopic Diagnosis: Histological type: Serous adenocarcinoma (Both fallopian tubes), Histological grade: Well differentiated. Involvement of another organ: Both cervix and uterus-free. One of ovaries is involved by the tumor, other ovary free. Lymph-vascular invasion: Not identified. Tumor necrosis: Not identified. Additional findings: Cervix reveals feature of chronic cervicitis. Endometrium shows non-secretory changes, Myometrium is unremarkable.) (if at all necessary?)

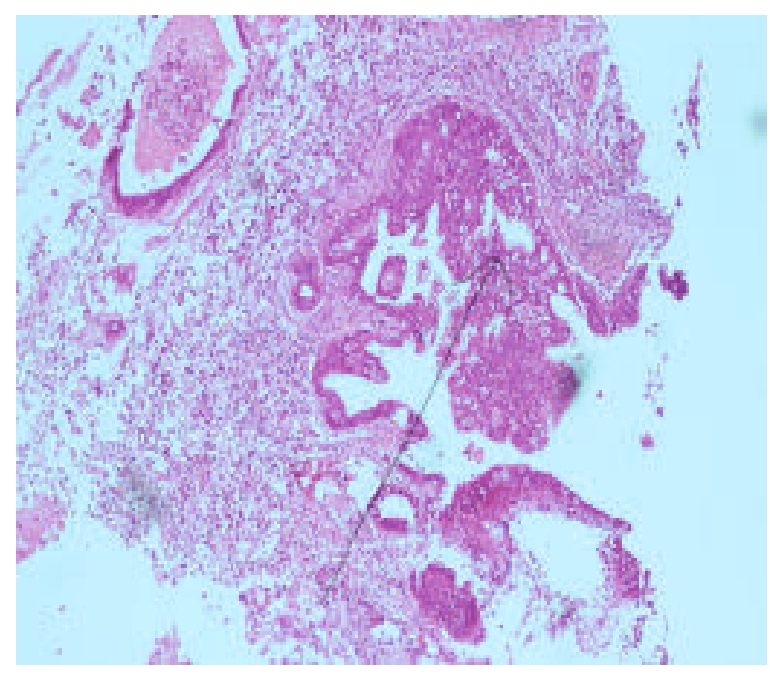

Histology in figure 1: adenocarcinoma of fallopian tube arising from epithelium. 


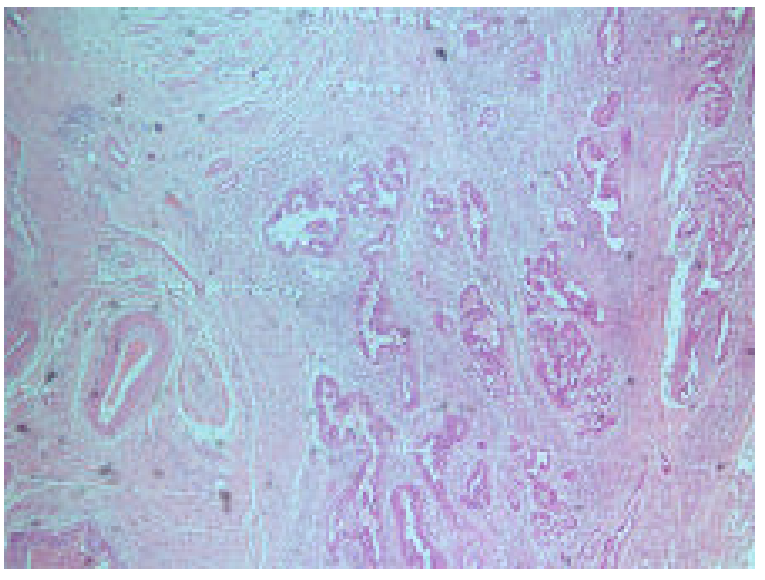

Figure 2-shows histological findings - well differentiated adenocarcinoma of fallopian tube.

\section{Discussion:}

Primary Fallopian tube carcinoma is uncommon and represents $<1 \%$ of all gynecologic malignancy ${ }^{3}$. Our case is a bilateral primary fallopian tube carcinoma. According to Markman et al (2000) bilateral involvement is reported only in $20 \%$ of patients ${ }^{7}$.

PFTC is common in post-menopausal women. Our patient is pre-menopausal and she is 42 years old. According to kalampoks et al (2013), the most common age of presentation is between 40 and 65 years, with a mean age of 55 years $^{8}$ but another study showed age range 21 to 78 years 9 .

The most common clinical presentation of primary fallopian tube carcinoma is nonspecific pelvic pain (37.5\%), abnormal vaginal bleeding (31.2\%), pelvic mass (18.8\%), and gastrointestinal symptoms $(12.5 \%)^{10}$. Latzko's triad of typical symptoms consists of intermittent profuse serosanguinous vaginal discharge, colicky pain relieved by discharge, and abdominal or pelvic mass and this triad was reported in only $15 \%$ of PFTC cases ${ }^{11}$. None of these symptoms are specific. Hydropstubaeprofluens, a pathognomonic feature, implies intermittent discharge of clear or bloody fluid either spontaneously or on pressure followed by shrinkage of an adnexal mass, but this feature is seen in only $5 \%$ of patients ${ }^{12}$. Our patient presented with lower abdominal pain and adnexal mass. But these are not specific for PFTC. She was diagnosed as a case of PID, which is found to be associated with PFTC. It is postulated that some factors increase the risk of this cancer, such as infertility, chronic tubal inflammation, endometriosis, nulliparity, smoking, and genetic predisposition $^{13}$.

In our case we could not make the proper diagnosis. We did not arrange frozen section biopsy as malignancy was not suspected. The diagnosis was first appreciated by the pathologist. In most cases, the preoperative diagnosis of PFTC is rare ${ }^{14,15}$. It is due to rarity and silent course of the disease. Intra operative diagnosis is also missed. Meng et al. found that intraoperative diagnosis is missed in up to $50 \%$ of patients and PFTC is usually confirmed by a pathologist. ${ }^{16}$ As the fallopian tube carcinoma is rare, the patient had a long history of PID and her symptoms \& signs were nonspecific - - it prompted us to make the diagnosis PID. In this case ultrasound scan revealed a cystic mass with solid regions that was not specific. But MRI was not performed and we did not consider frozen section biopsy. All these led us to misdiagnose the case.

The most common histological type of PFTC is papillary serous carcinoma. Histologically it resembles serous ovarian adenocarcinoma. Because it is difficult to differentiate PFTC from epithelial ovarian cancer (EOC) diagnostic criteria for the diagnosis of PFTC should include the following ${ }^{17}$

- The main tumor is in the tube and arises from the endosalpinx;

- Histologically, the pattern reproduces the epithelium of the mucosa;

- Transition between benign and malignant epithelium should be demonstrable; and

- The ovaries and endometrium are either normal or have a smaller tumor volume than that of the tube.

Recently, Singh et al proposed that the identification of tumor inside the fallopian tubes, even in the presence of larger tumors in other location, supports the diagnosis of PFTC ${ }^{18}$.

Surgery is the cornerstone of the therapy for PFTC. Surgical staging and management are similar to that for epithelial ovarian carcinoma. Surgical procedure includes intact removal of tumor, total hysterectomy with removal of fallopian tubes and ovaries, infracolic omentectomy, biopsies from abdominal peritoneal implants, pelvic and para-aortic lymph node biopsies. Our patient did not receive optimal surgery as she had been incidentally detected during routine histological examination. 


\section{Conclusion:}

PFTC should be considered in differential diagnosis of pre and postmenopausal women who present with pelvic pain, adnexal mass or complicated pelvic inflammatory disease. MRI should be considered as pre-operative work up. MRI can identify the characteristic features of PFTC and it is helpful in detection and in differentiating PFTC from other pelvic masses.

\section{Acknowledgement:}

We would like to thank Col. (Dr.) Jaynul Islam, AFIP, Dhaka and Dr. Shahnaj Zahan, CBMC, Mymensingh for their guidance regarding histological findings.

\section{Reference:}

1. Sedlis A, Carcinoma of the fallopian tube. Surg Clin North Am. 1978, Feb;58(1):121-9.

2. Onal MM, HanhanM ,P0lanci B , Tinar T, Fallopian tube malignancies: experience of Social Security Agency Aegean Maternity Hospital. Int. J Gynecol Cancer,2004; 14: 595-9.

3. Riska A, Leminen A, Pukkala E. Sociodemographic determinants of incidence of primary fallopian tube carcinoma, Finland 1953-97. Int J Cancer. 2003; 104:643645.

4. Aziz S, Kuperstein G, Rosen B, Cole D, Nedelcu R, McLaughlin J, Narod SA. . A genetic epidemiological study of carcinoma of the fallopian tube. Gynecol Oncol. 2001 Mar;80(3):341-5.

5. Anthoulakis C, Nikoloudis N. Pelvic MRI as the "gold standard" in the subsequent evaluation of ultrasoundindeterminate adnexal lesions: a systematic review. Gynecol Oncol. 2014; 132:661-668.

6. Ma FH, Cai SQ, Qiang JW, Zhao SH, Zhang GF, Rao YM. MRI for differentiating primary fallopian tube carcinoma from epithelial ovarian cancer. J MagnReson Imaging. 2015 Jul;42(1):42-7.

7. Markman M, Zaino RJ, Fleming PA, Barakat RR. Carcinoma of the fallopian tube.IN: Hoskins WJ, Perez CA,
Young RC, eds. Principles and practice of gynecologic oncology. Philadelphia, Pa: Lippincott Williams \& Wilkins, 2000; 1099-1112.

8. Kalampokas E, Kalampokas T, Tourountous I. Primary fallopian tube carcinoma. Eur J ObstetGynecolReprod Biol. 2013; 169(2):155-161.

9. Yoonessi M. Carcinoma of the fallopian tube. ObstetGynecolSurv. 1979; 34:257-268

10. Hei-YuLau, Yi-JEnChen, Ming-Shyen Yen, Ru-FenChen, Shu-O Yeh, Nae-Fang Twu.Primary fallopian tube carcinoma: A clinic pathologic analysis and literature review. Journal of the Chinese Medical Association. October 2013; 76(10):583-587.

11. Ajithkumar TV, Minimole AL, John MM. Primary fallopian tube carcinoma.ObstetGynecol Surv,2005; 60:247-252.

12. Honoré LH. Pathology of the fallopian tube and broad ligament. In: Fox H, Wells M. Haines and Taylor obstetrical \& gynecologic pathology, 5th Ed. New York, NY: Churchill Livingstone, 2003: 605-615.

13. I. Alvarado-Cabrero, R.H. Young, E.C. Vamvakas, R.E. Scully Carcinoma of the fallopian tube: a clinico pathological study of 150 cases with observation on staging and prognostic factors Gynecol Oncol, 1999; 72: 367-379.

14. Boutselis JG, Thompson JN. Clinical aspects of primary carcinoma of the Fallopian tube: a clinical study of 14 cases. Am J ObstetGynecol 1971; 111:98-101.

15. Pfeiffer P, Mogensen H, Amtrup F et al. Primary carcinoma of the fallopian tube. A retrospective study of patients reported to the Danish Cancer Registry in a five-year period. Acta Oncol 1989; 28:7-11.

16. Meng ML, Gan-Gao, Scheng-Sun et al. Diagnosis of primary adenocarcinoma of the fallopian tube. J Cancer Res Clinncer Res Clin Oncol 1985; 110:136-140.

17. Hu CY, Taymor ML, Hertig AT. Primary carcinoma of the fallopian tube. Am J ObstetGynecol 1950 Jan; 59:58-67.

18. Singh N, Gilks CB, Wilkinson N, McCluggage WG. Assignment of primary site in high-grade serous tubal, ovarian and peritoneal carcinoma: a proposal. Histopathology. 2014; 65:149-154. 